Article

\title{
Existence Results for Block Matrix Operator of Fractional Orders in Banach Algebras
}

\author{
Hind Hashem ${ }^{1,2, *(\mathbb{D}}$, Ahmed El-Sayed ${ }^{1}$ and Dumitru Baleanu ${ }^{3,4}(\mathbb{D}$ \\ 1 Faculty of Science, Alexandria University, Alexandria 21526, Egypt \\ 2 Faculty of Science, Qassim University, Buraidah 51452, Saudi Arabia \\ 3 Department of Mathematics, Cankaya University, Balgat, 06530 Ankara, Turkey \\ 4 Institute of Space Sciences, 077125 Magurele-Bucharest, Romania \\ * Correspondence: 3922@qu.edu.sa
}

Received: 14 May 2019; Accepted: 15 August 2019; Published: 17 September 2019

check for updates

\begin{abstract}
This paper is concerned with proving the existence of solutions for a coupled system of quadratic integral equations of fractional order in Banach algebras. This result is a direct application of a fixed point theorem of Banach algebras. Some particular cases, examples and remarks are illustrated. Finally, the stability of solutions for that coupled system are studied.
\end{abstract}

Keywords: fractional order operators; quadratic integral equations; coupled system; $2 \times 2$ block operator matrix

MSC: Primary 26A33; Secondary 45D05; 60G22; 33E30

\section{Introduction and Preliminaries}

Operators which have an operator matrix representation occur in various fields such as system theory, quantum mechanics, hydrodynamics and magnetohydro-dynamics (see [1-3]).

According to their origin, they may have rather different structure, and their study may require quite different approaches.

Let $\mathbb{A}$ be an operator which has the form

$$
\mathbb{A}=\left(\begin{array}{cc}
T_{1} & T_{2} \cdot T_{2}^{\prime} \\
T_{3} & T_{4}
\end{array}\right)
$$

where $T_{1}, T_{2}, T_{2}^{\prime}, T_{3}, T_{4}$ are nonlinear operators defined on Banach algebras. This kind of operators is studied by many researchers [4-6].

Amar and et al. [7] introduced and studied a coupled system of differential equations under boundary conditions of Rotenberg's model type, the last one arising in growing cell populations. The entries of block operator matrix associated to this system are nonlinear and act on the Banach space.

Kaddachi and et al. [4] concentrated on answering the question: Under which conditions on its entries does the $2 \times 2$ operator matrix (Equation (1)) acting on a product of Banach algebras has a fixed point? In [4], some fixed point theorems of a $2 \times 2$ block operator matrix defined on nonempty bounded closed convex subsets of Banach algebras are studied, where the entries are nonlinear operators. Furthermore, the obtained results are applied to a coupled system of nonlinear equations. 
Let $X=\mathbb{C}(I, \mathbb{R}), I=[0, b]$ and $\alpha, \beta>0$. In this work, the following coupled system of fractional order

$$
\begin{aligned}
& v(t)=f_{1}(t, v(t))+g_{1}(t, w(t)) \int_{0}^{t} \frac{(t-s)^{\alpha-1}}{\Gamma(\alpha)} u_{1}(s, w(s)) d s, t \in I, \\
& w(t)=f_{2}(t, w(t))+g_{2}(t, v(t)) \int_{0}^{t} \frac{(t-s)^{\beta-1}}{\Gamma(\beta)} u_{2}(s, v(s)) d s, t \in I .
\end{aligned}
$$

is studied in Banach algebras; some particular cases are given; and some examples and remarks are illustrated. Finally, the stability of solutions for the coupled system in Equation (2) is studied.

The solution of Equation (2) may be defined by a vector function $\left(\begin{array}{c}v \\ w\end{array}\right) \in X \times X$ that satisfies (2).

Now, we introduce the following definitions of fractional operators.

Definition 1 ([8]). The Riemann-Liouville fractional integral of order $\beta>0$ of the function $f:[a, b] \rightarrow \mathbb{R}$ is given by

$$
I_{a}^{\beta} f(t)=\int_{a}^{t} \frac{(t-s)^{\beta-1}}{\Gamma(\beta)} f(s) d s, t>a
$$

and when $a=0$, we have $I^{\beta} f(t)=I_{0}^{\beta} f(t), t>0$.

Definition 2 ([8]). The Riemann-Liouville fractional derivative of order $\alpha \in(0,1)$ of a function $f$ is defined as

$$
{ }_{R} D_{a}^{\alpha} f(t)=\frac{d}{d t} \int_{a}^{t} \frac{(t-s)^{-\alpha}}{\Gamma(1-\alpha)} f(s) d s, \quad t \in[a, b]
$$

or

$$
{ }_{R} D_{a}^{\alpha} f(t)=\frac{d}{d t} I_{a}^{1-\alpha} f(t), t \in[a, b]
$$

\section{Existence Theorem}

Coupled systems of integral and differential equations are studied in many papers [9-13].

Especially, the investigation for coupled systems of fractional differential equations appears in many studies (e.g., [9,11,14-17]).

Assume that

(i) $\quad u_{j}: I \times \mathbb{R} \rightarrow \mathbb{R}, j=1,2$ satisfies the Carathéodory condition and

$$
\left|u_{j}(s, v)\right| \leq m_{j}(s) \in L^{1}[I] \quad \forall(s, v) \in I \times \mathbb{R},
$$

$k_{j}=\max _{s \in I} I^{\gamma_{j}} m_{j}(s)$ for any $\gamma_{1} \leq \alpha$ and $\gamma_{2} \leq \beta$.

(ii) $f_{j}, g_{j}: I \times \mathbb{R} \rightarrow \mathbb{R}$ are continuous and

$M_{j}=\max _{(s, v) \in I \times \mathbb{R}}\left|f_{j}(s, v)\right|, j=1,2 \quad N_{j}=\max _{(s, v) \in I \times \mathbb{R}}\left|g_{j}(s, v)\right|, j=1,2$ respectively.

(iii) There exist constants $l_{j}$ and $h_{j}$, which satisfy

$$
\left|f_{j}(s, v)-f_{j}(s, w)\right| \leq l_{j}|v-w|, \quad j=1,2
$$

and

$$
\left|g_{j}(s, v)-g_{j}(s, w)\right| \leq h_{j}|v-w|, \quad j=1,2
$$


$\forall s \in I$ and $v, w \in \mathbb{R}$.

Theorem 1. Let Assumptions (i)-(iii) be satisfied. Moreover, if $h_{1} k_{1} h_{2} k_{2}<\left(1-l_{1}\right)\left(1-l_{2}\right) \Gamma\left(\alpha-\gamma_{1}+1\right) \Gamma(\beta-$ $\left.\gamma_{2}+1\right)$, then the exists at least one solution for Equation (2) in $X \times X$.

Proof. Consider the operators $T_{1}, T_{2}, T_{3}, T_{4}$ and $T_{2}^{\prime}$ on $X$ defined by:

$$
\left\{\begin{array}{l}
\left(T_{1} v\right)(t)=f_{1}(t, v(t)) \\
\left(T_{2} w\right)(t)=g_{1}(t, w(t)) \\
\left(T_{3} w\right)(t)=g_{2}(t, w(t)) \int_{0}^{t} \frac{(t-s)^{\beta-1}}{\Gamma(\beta)} u_{2}(s, w(s)) d s \\
\left(T_{4} v\right)(t)=f_{2}(t, v(t)) \\
\left(T_{2}^{\prime} w\right)(t)=\int_{0}^{t} \frac{(t-s)^{\alpha-1}}{\Gamma(\alpha)} u_{1}(s, w(s)) d s
\end{array}\right.
$$

The coupled system in Equation (2) may have the form:

$$
\left\{\begin{array}{l}
v(t)=T_{1} v(t)+T_{2} w(t) \cdot T_{2}^{\prime} w(t) \\
w(t)=T_{4} w(t)+T_{3} v(t)
\end{array}\right.
$$

and

$$
\left(\begin{array}{c}
v \\
w
\end{array}\right)=\left(\begin{array}{cc}
T_{1} & T_{2} \cdot T_{2}^{\prime} \\
T_{3} & T_{4}
\end{array}\right) \cdot\left(\begin{array}{c}
v \\
w
\end{array}\right) .
$$

Define

$$
\begin{gathered}
S=\left\{v \in X,\|v\| \leq M_{1}+\frac{N_{1} k_{1} b^{\alpha-\gamma_{1}}}{\Gamma\left(\alpha-\gamma_{1}+1\right)}\right\} \\
S^{\prime}=\left\{w \in X,\|w\| \leq M_{2}+\frac{N_{2} k_{2} b^{\beta-\gamma_{2}}}{\Gamma\left(\beta-\gamma_{2}+1\right)}\right\} .
\end{gathered}
$$

For, let $v_{1}, v_{2} \in S$. Thus,

$$
\left\|T_{1} v_{1}(t)-T_{1} v_{2}(t)\right\| \leq l_{1}\left\|v_{1}-v_{2}\right\|
$$

and

$$
\left\|T_{2} v_{1}(t)-T_{2} v_{2}(t)\right\| \leq h_{1}\left\|v_{1}-v_{2}\right\|
$$

In addition, set

$$
T_{3} v_{1}(t)=G v_{1}(t) \cdot U v_{1}(t)=(G \cdot U) v_{1}(t)
$$

where $G v_{1}(t)=g_{2}\left(t, v_{1}(t)\right)$ and $U v_{1}(t)=I^{\beta} u_{2}\left(t, v_{1}(t)\right)$

$$
\begin{aligned}
\left\|T_{3} v_{1}(t)-T_{3} v_{2}(t)\right\| & =\left\|(G \cdot U) v_{1}(t)-(G \cdot U) v_{2}(t)\right\| \\
& \leq\left\|U v_{1}(t)\right\| \cdot\left\|G v_{1}(t)-G v_{2}(t)\right\| \\
& \leq \frac{k_{2} h_{2} b^{\beta-\gamma_{2}}}{\Gamma\left(\beta-\gamma_{2}+1\right)} \cdot\left\|v_{1}-v_{2}\right\| .
\end{aligned}
$$

Furthermore,

$$
\begin{aligned}
\left|T_{3} w(t)\right| & \leq\left|g_{2}(t, w(t))\right| \int_{0}^{t} \frac{(t-s)^{\beta-1}}{\Gamma(\beta)}\left|u_{2}(s, w(s))\right| d s \\
& \leq \frac{N_{2} k_{2} b^{\beta-\gamma_{2}}}{\Gamma\left(\beta-\gamma_{2}+1\right)}
\end{aligned}
$$


for each $t_{1}, t_{2} \in I$ and $t_{1}<t_{2}$, we get

$$
\begin{aligned}
\left|\left(T_{3} w\right)\left(t_{2}\right)-\left(T_{3} w\right)\left(t_{1}\right)\right| & =\mid g_{2}\left(t_{2}, w\left(t_{2}\right)\right) I^{\beta} u_{2}\left(t_{2}, w\left(t_{2}\right)\right)-g_{2}\left(t_{1}, w\left(t_{1}\right)\right) I^{\beta} u_{2}\left(t_{1}, w\left(t_{1}\right)\right) \\
& +g_{2}\left(t_{1}, w\left(t_{1}\right)\right) I^{\beta} u_{2}\left(t_{2}, w\left(t_{2}\right)\right)-g_{2}\left(t_{1}, w\left(t_{1}\right)\right) I^{\beta} u_{2}\left(t_{2}, w\left(\left(t_{2}\right)\right) \mid\right. \\
& \leq\left|g_{2}\left(t_{2}, w\left(t_{2}\right)\right)-g_{2}\left(t_{1}, w\left(t_{1}\right)\right)\right| I^{\beta}\left|u_{2}\left(t_{2}, w\left(t_{2}\right)\right)\right| \\
& +\left|g\left(t_{1}, w\left(t_{1}\right)\right)\right|\left|I^{\beta} u_{2}\left(t_{2}, w\left(t_{2}\right)\right)-I^{\beta} u_{2}\left(t_{1}, w\left(t_{1}\right)\right)\right|,
\end{aligned}
$$

but

$$
\left|I^{\beta} u_{2}\left(t_{2}, w\left(t_{2}\right)\right)-I^{\beta} u_{2}\left(t_{1}, w\left(t_{1}\right)\right)\right| \leq \int_{t_{1}}^{t_{2}} \frac{\left(t_{2}-s\right)^{\beta-1}}{\Gamma(\beta)}\left|u_{2}(s, w(s))\right| d s
$$

Then,

$$
\left|I^{\beta} u_{2}\left(t_{2}, w\left(t_{2}\right)\right)-I^{\beta} u_{2}\left(t_{1}, w\left(t_{1}\right)\right)\right| \leq k_{2} \frac{\left(t_{2}-t_{1}\right)^{\beta-\gamma_{2}}}{\Gamma\left(\beta-\gamma_{2}+1\right)} .
$$

Then, we get

$$
\left|\left(T_{3} w\right)\left(t_{2}\right)-\left(T_{3} w\right)\left(t_{1}\right)\right| \leq \frac{k_{2} h_{2} b^{\beta-\gamma_{2}}}{\Gamma\left(\beta-\gamma_{2}+1\right)}\left|w\left(t_{2}\right)-w\left(t_{1}\right)\right|+\frac{k_{2} N_{2}}{\Gamma\left(\beta-\gamma_{2}+1\right)}\left(t_{2}-t_{1}\right)^{\beta-\gamma_{2}}
$$

Then, $\overline{U S^{\prime}}$ is relatively compact.

We prove that $T_{3}(S) \subseteq\left(I-T_{4}\right)\left(S^{\prime}\right)$, for $v_{1} \in S$.

Now, we can introduce a function $\phi_{v_{1}}: X \rightarrow X$ by

$$
w \rightarrow T_{3} v_{1}+T_{4} v_{2}
$$

then the function $\phi_{v_{1}}$ is a contraction with a constant $l_{2}+\frac{h_{2} k_{2} b^{\beta-\gamma_{2}}}{\Gamma\left(\beta-\gamma_{2}+1\right)}$. Then, there exists a unique point $w \in X$ where $T_{3} v+T_{4} w=w$ implies $T_{3} v=\left(I-T_{4}\right) w$. Thus,

$$
T_{3}(s) \subseteq\left(I-T_{4}\right) X
$$

For $w \in X, \exists s^{*} \in I$ such that

$$
\begin{aligned}
\|w\|_{\infty}=\left|w\left(s^{*}\right)\right| & =\left|T_{3} v\left(s^{*}\right)+T_{4} w\left(s^{*}\right)\right| \\
& \leq\left|g_{2}\left(s^{*}, v\left(s^{*}\right)\right) I^{\beta} u_{2}\left(s^{*}, v\left(s^{*}\right)\right)+f_{2}\left(s^{*}, w\left(s^{*}\right)\right)\right| \\
& \leq \frac{N_{2} k_{2} b^{\beta-\gamma_{2}}}{\Gamma\left(\beta-\gamma_{2}+1\right)}+M_{2} .
\end{aligned}
$$

Then, $T_{3}(S) \subseteq\left(I-T_{4}\right)\left(S^{\prime}\right)$.

For $v \in S^{\prime}$, then

$$
\begin{aligned}
\left|T_{2}^{\prime} v\left(t_{n}\right)-T_{2}^{\prime} v(t)\right| & =\left|I^{\alpha} u_{1}\left(t_{n}, v\left(t_{n}\right)\right)-I^{\alpha} u_{1}(t, v(t))\right| \\
& \leq \frac{k_{1}}{\Gamma\left(\alpha-\gamma_{1}+1\right)}\left|t_{n}-t\right|
\end{aligned}
$$

since $t_{n} \rightarrow t$ and $u_{1}$ is continuous in the second argument, then by Lebesgue Dominated Convergence Theorem, we have

$$
u_{1}\left(t_{n}, v\left(t_{n}\right)\right) \rightarrow u_{1}(t, v(t)) \text { in } \mathbb{R} \Rightarrow T_{2}^{\prime} v\left(t_{n}\right) \rightarrow T_{2}^{\prime} v(t) \text { in } \mathbb{R}
$$

thus $T_{2}^{\prime} v \in \mathbb{C}(I, \mathbb{R})$. 
Defining $T^{\prime}=T_{2}^{\prime}\left(1-T_{4}\right)^{-1} T_{3}$, Assumption (ii) implies that

$$
\begin{aligned}
M & =\left\|T^{\prime}(S)\right\|=\sup _{w \in S}\left|T^{\prime}(w)\right| \\
& \leq \sup _{t \in I}\left|\int_{0}^{t} \frac{(t-s)^{\alpha-1}}{\Gamma(\alpha)} u_{1}(s, w(s)) d s\right| \\
& \leq \frac{k_{1} b^{\alpha-\gamma_{1}}}{\Gamma\left(\alpha-\gamma_{1}+1\right)}
\end{aligned}
$$

and therefore $h_{1} k_{1} h_{2} k_{2}<\left(1-l_{1}\right)\left(1-l_{2}\right) \Gamma\left(\alpha-\gamma_{1}+1\right) \Gamma\left(\beta-\gamma_{2}+1\right)$.

Let $v_{1}, v_{2} \in S$, then $\forall s \in I$. We obtain

$$
\left|T_{1} v_{1}(s)+T_{2}\left(I-T_{4}\right)^{-1} T_{3} v_{1}(s) T_{2}^{\prime}\left(I-T_{4}\right)^{-1} T_{3} v_{2}\right| \leq M_{1}+\frac{N_{1} k_{1} b^{\alpha-\gamma_{1}}}{\Gamma\left(\alpha-\gamma_{1}+1\right)} .
$$

This implies that

$$
T_{1} v_{1}+T_{2}\left(I-T_{4}\right)^{-1} T_{3} v_{1} T_{2}^{\prime}\left(I-T_{4}\right)^{-1} T_{3} v_{2} \in S \text { for any } v_{1}, v_{2} \in S .
$$

Now, all conditions of Theorem 4.2 in [4] are verified and our results follows.

Example 1. Let $I=[0,1]$. Consider the fractional order coupled system

$$
\begin{aligned}
& v(t)=t \sin \left(\frac{v(t)}{4}\right)+\frac{w(t)}{3+t} I^{3 / 2} \frac{2 t w(t)}{1+w(t)}, \quad t \in I \\
& w(t)=\frac{t+\sin \left(\frac{w(t)}{2}\right)}{2+t^{2}}+\frac{v(t)}{4} I^{2 / 3} \frac{v(t)}{1+v(t)}, \quad t \in I .
\end{aligned}
$$

Set

$$
\begin{gathered}
f_{1}(s, v)=s \sin \left(\frac{v(s)}{4}\right), \quad u_{1}(s, w)=\frac{2 s w(s)}{1+w(s)}, \quad g_{1}(s, w)=\frac{w(s)}{3+s^{\prime}} \\
f_{2}(s, w)=\frac{s+\sin \left(\frac{w(s)}{2}\right)}{2+s^{2}}, \quad u_{2}(s, v)=\frac{v(s)}{1+v(s)}, \quad g_{2}(s, w)=\frac{w(s)}{4} .
\end{gathered}
$$

Then, we easily get

- $\left|u_{1}(s, v)\right| \leq 2 s=m_{1}(s)$ and $\left|u_{2}(s, v)\right| \leq 1=m_{2}(s)$.

Choose $\gamma_{1}=\gamma_{2}=1 / 2$, then we can obtain $k_{1}=\frac{8}{3 \sqrt{\pi}}$ and $k_{2}=\frac{2}{\sqrt{\pi}}$

$$
\begin{aligned}
\left|g_{1}\left(t, w_{1}\right)-g_{1}\left(t, w_{2}\right)\right| & \leq \frac{1}{3}\left|w_{1}-w_{2}\right| \\
\left|g_{2}\left(t, v_{1}\right)-g_{2}\left(t, v_{2}\right)\right| & \leq \frac{1}{4}\left|v_{1}-v_{2}\right|
\end{aligned}
$$

and

$$
\left|f_{i}\left(t, v_{1}\right)-f_{i}\left(t, v_{2}\right)\right| \leq \frac{1}{2}\left|v_{1}-v_{2}\right|, \quad i=1,2
$$

Then, the inequality $h_{1} k_{1} h_{2} k_{2}<\left(1-l_{1}\right)\left(1-l_{2}\right) \Gamma(\alpha-\gamma+1) \Gamma(\beta-\gamma+1)$ is verified. 


\section{Stability of Solutions of the Coupled System}

Here, asymptotic stability on $\mathbb{R}_{+}$of the solution $z=(v, w)$ of the coupled system in Equation (2) is studied.

Definition 3. A pair $z_{1}=\left(v_{1}, w_{1}\right)$ is said to be an asymptotically stable solution of Equation (2) if for any $\varepsilon>0$ there exists $T^{\prime}=T^{\prime}(\varepsilon)>0$ such that for very $t \geq T^{\prime}$ and for every other solution $z_{2}=\left(v_{2}, w_{2}\right)$ of $(2)$,

$$
\left|z_{1}(t)-z_{2}(t)\right| \leq \varepsilon
$$

Given two solutions $z_{1}$ and $z_{2}$ of Equation (2), then we have

$$
\begin{aligned}
\left|v_{1}(t)-v_{2}(t)\right| & \leq\left|f_{1}\left(t, v_{1}(t)\right)-f_{1}\left(t, v_{2}(t)\right)\right| \\
& +\mid g_{1}\left(t, w_{1}(t)\right) \int_{0}^{t} \frac{(t-s)^{\alpha-1}}{\Gamma(\alpha)} u_{1}\left(s, w_{1}(s)\right) d s \\
& -g_{1}\left(t, w_{2}(t)\right) \int_{0}^{t} \frac{(t-s)^{\alpha-1}}{\Gamma(\alpha)} u_{1}\left(s, w_{2}(s)\right) d s \mid \\
& \leq l_{1}\left|v_{1}(t)-v_{2}(t)\right| \\
& +g_{1}\left(t, w_{1}(t)\right)\left|\int_{0}^{t} \frac{(t-s)^{\alpha-1}}{\Gamma(\alpha)}\right| u_{1}\left(s, w_{1}(s)\right)-u_{1}\left(s, w_{2}(s)\right) \mid d s \\
& +\left|g_{1}\left(t, w_{2}(t)\right)-g_{1}\left(t, w_{1}(t)\right)\right| \int_{0}^{t} \frac{(t-s)^{\alpha-1}}{\Gamma(\alpha)}\left|u_{1}\left(s, w_{2}(s)\right)\right| d s \\
& \leq l_{1}\left|v_{1}(t)-v_{2}(t)\right|+2\left|g_{1}\left(t, w_{1}(t)\right)\right| \int_{0}^{t} \frac{(t-s)^{\alpha-1}}{\Gamma(\alpha)} m_{1}(s) d s \\
& +\left|g_{1}\left(t, w_{2}(t)\right)-g_{1}\left(t, w_{1}(t)\right)\right| \int_{0}^{t} \frac{(t-s)^{\alpha-1}}{\Gamma(\alpha)} m_{1}(s) d s \\
& \leq l_{1}\left|v_{1}(t)-v_{2}(t)\right|+\frac{2 k_{1} N_{1} b^{\alpha-\gamma}}{\Gamma\left(\alpha-\gamma_{1}+1\right)}+\frac{h_{1} k_{1} b^{\alpha-\gamma}}{\Gamma\left(\alpha-\gamma_{1}+1\right)}\left|w_{1}-w_{2}\right|,
\end{aligned}
$$

then

$$
\left(1-l_{1}\right)\left\|v_{1}(t)-v_{2}(t)\right\| \leq \frac{2 k_{1} N_{1} b^{\alpha-\gamma_{1}}}{\Gamma\left(\alpha-\gamma_{1}+1\right)}+\frac{h_{1} k_{1} b^{\alpha-\gamma_{1}}}{\Gamma\left(\alpha-\gamma_{1}+1\right)}\left\|w_{1}-w_{2}\right\| .
$$

In the same fashion, we obtain

$$
\left(1-l_{2}\right)\left\|w_{1}(t)-w_{2}(t)\right\| \leq \frac{2 k_{2} N_{2} b^{\beta-\gamma_{2}}}{\Gamma\left(\beta-\gamma_{2}+1\right)}+\frac{h_{2} k_{2} b^{\beta-\gamma_{2}}}{\Gamma\left(\beta-\gamma_{2}+1\right)}\left\|v_{1}-v_{2}\right\|
$$

and

$\left[1-l_{1}-\frac{h_{2} k_{2} b^{\beta-\gamma_{2}}}{\Gamma\left(\beta-\gamma_{2}+1\right)}\right]\left\|v_{1}-v_{2}\right\|+\left[1-l_{2}-\frac{h_{1} k_{1} b^{\alpha-\gamma_{1}}}{\Gamma\left(\alpha-\gamma_{1}+1\right)}\right]\left\|w_{1}-w_{2}\right\| \leq \frac{2 k_{1} N_{1} b^{\alpha-\gamma_{1}}}{\Gamma\left(\alpha-\gamma_{1}+1\right)}+\frac{2 k_{2} N_{2} b^{\beta-\gamma_{2}}}{\Gamma\left(\beta-\gamma_{2}+1\right)}$

Let $\Lambda=\min \left\{1-l_{1}-\frac{h_{2} k_{2} b^{\beta-\gamma_{2}}}{\Gamma\left(\beta-\gamma_{2}+1\right)}, 1-l_{2}-\frac{h_{1} k_{1} b^{\alpha-\gamma_{1}}}{\Gamma\left(\alpha-\gamma_{1}+1\right)}\right\}$, then

$$
\left\|v_{1}-v_{2}\right\|+\left\|w_{1}-w_{2}\right\| \leq \Lambda^{-1}\left[\frac{2 k_{1} N_{1} b^{\alpha-\gamma_{1}}}{\Gamma\left(\alpha-\gamma_{1}+1\right)}+\frac{2 k_{2} N_{2} b^{\beta-\gamma_{2}}}{\Gamma\left(\beta-\gamma_{2}+1\right)}\right]
$$

Since

$$
z_{1}(t)-z_{2}(t)=\left(v_{1}(t)-v_{2}(t), w_{1}(t)-w_{2}(t)\right)
$$


then

$$
\begin{aligned}
\left\|z_{1}(t)-z_{2}(t)\right\| & \leq\left\|v_{1}(t)-v_{2}(t)\right\|+\left\|w_{1}(t)-w_{2}(t)\right\| \\
& \leq \Lambda^{-1}\left[\frac{2 k_{1} N_{1} b^{\alpha-\gamma}}{\Gamma\left(\alpha-\gamma_{1}+1\right)}+\frac{2 k_{2} N_{2} b^{\beta-\gamma_{2}}}{\Gamma\left(\beta-\gamma_{2}+1\right)}\right] \\
& \leq \varepsilon
\end{aligned}
$$

Then, we obtain the following theorem.

Theorem 2. Let assumptions of Theorem 1 be satisfied,

$$
l_{1} \Gamma\left(\beta-\gamma_{2}+1\right)+h_{2} k_{2} b^{\beta-\gamma_{2}}<\Gamma\left(\beta-\gamma_{2}+1\right), l_{2} \Gamma\left(\alpha-\gamma_{1}+1\right)+h_{1} k_{1} b^{\alpha-\gamma_{1}}<\Gamma\left(\alpha-\gamma_{1}+1\right),
$$

and

$$
\Lambda^{-1}\left[\frac{2 k_{1} N_{1} b^{\alpha-\gamma_{1}}}{\Gamma\left(\alpha-\gamma_{1}+1\right)}+\frac{2 k_{2} N_{2} b^{\beta-\gamma_{2}}}{\Gamma\left(\beta-\gamma_{2}+1\right)}\right] \leq \varepsilon
$$

Then, the solution of Equation (2) is asymptotically stable on $\mathbb{R}_{+}$.

\section{Further Results}

Consequently, we have the following results in $X \times X$.

(i) Letting $\alpha, \beta \rightarrow 1$, then we have the coupled system of quadratic integral equations

$$
\begin{aligned}
v(t) & =f_{1}(t, v(t))+g_{1}(t, w(t)) \int_{0}^{t} u_{1}(s, w(s)) d s, \\
w(t) & =f_{2}(t, w(t))+g_{2}(t, v(t)) \int_{0}^{t} u_{2}(s, v(s)) d s .
\end{aligned}
$$

(ii) Letting $g_{1}=g_{2}=0$, we get the coupled system of functional equations

$$
\begin{aligned}
v(t) & =f_{1}(t, v(t)) \\
w(t) & =f_{2}(t, w(t)) .
\end{aligned}
$$

(iii) Putting $f_{1}(t, w)=a_{1}(t), f_{2}(t, v)=a_{2}(t)$, then we have the coupled system of quadratic integral equations of fractional order

$$
\begin{aligned}
& v(t)=a_{1}(t)+g_{1}(t, w(t)) \int_{0}^{t} \frac{(t-s)^{\alpha-1}}{\Gamma(\alpha)} u_{1}(s, w(s)) d s \\
& w(t)=a_{2}(t)+g_{2}(t, v(t)) \int_{0}^{t} \frac{(t-s)^{\beta-1}}{\Gamma(\beta)} u_{2}(s, v(s)) d s .
\end{aligned}
$$

(v) Letting $f_{1}(t, w)=a_{1}(t), f_{2}(t, v)=a_{2}(t)$, then we get the coupled system of fractional integral equations

$$
\begin{aligned}
v(t) & =a_{1}(t)+\int_{0}^{t} \frac{(t-s)^{\alpha-1}}{\Gamma(\alpha)} u_{1}(s, w(s)) d s \\
w(t) & =a_{2}(t)+\int_{0}^{t} \frac{(t-s)^{\beta-1}}{\Gamma(\beta)} u_{2}(s, v(s)) d s .
\end{aligned}
$$


System of Fractional Differential Equations

Let

$$
\begin{aligned}
& { }_{R} D^{\alpha} v(t)=u_{1}(t, w(t)), t \in J \text { and } v(0)=0, \quad 0<\alpha<1 \\
& { }_{R} D^{\beta} w(t)=u_{2}(t, v(t)), t \in J \text { and } w(0)=0, \quad 0<\beta<1
\end{aligned}
$$

where ${ }_{R} D^{\alpha}$ is a Riemann-Liouville fractional derivative of order $0<\alpha<1$.

Theorem 3. Let assumptions of Theorem 1 be satisfied. Then, there exists at least one solution for Equation (29) in $X \times X$.

The proof is straight forward as in [11].

By direct calculations, we can prove an existence result for the following coupled systems

$$
\begin{gathered}
v^{\prime}(t)=f_{1}(t, w(t)), \quad v(0)=v_{0} \\
w^{\prime}(t)=f_{2}(t, v(t)), \quad w(0)=w_{0} .
\end{gathered}
$$

\section{Conclusions}

The theory of block operator matrices opens up a new line of attack of mathematical problems. During the past years, several papers are devoted to the investigation of linear operator matrices defined by $2 \times 2$ block operator matrices (Equation (1)).

In this paper, we prove an existence theorem of solutions for a coupled system of quadratic integral equations of fractional order in Banach algebras, by a direct application of a block operator fixed point theorem [4]. This coupled system includes many key coupled systems of integral and differential equations that arise in nonlinear analysis and their applications. Some examples and remarks are illustrated. Finally, we study the stability of solutions for the coupled system in Equation (2).

Author Contributions: Conceptualization, H.H.G.; methodology, A.M.A. and D.B.; validation, H.H.G., A.M.A. and D.B.; formal analysis, H.H.G.; investigation, H.H.G.; writing-original draft preparation, A.M.A. and D.B.; writing-review and editing, A.M.A. and D.B.; visualization, A.M.A. and D.B.; supervision, H.H.G.; project administration, D.B.

Funding: This research received no external funding.

Conflicts of Interest: The authors declare no conflict of interest.

\section{References}

1. Bashir, A.; Nieto, J. Existence results for a coupled system of nonlinear fractional differential equations with three-point boundary conditions. Comput. Math. Appl. 2009, 58, 1838-1843.

2. Gao, X.; Yu, J. Synchronization of two coupled fractional-order chaotic oscillators. Chaos Solitons Fractals 2005, 26, 141-145. [CrossRef]

3. Saad, K.; Gómez-Aguilar, J.F.; Atangana, A.; Escobar-Jiménez, R.F. Model of Coupled System of Fractional Reaction-Diffusion Within a New Fractional Derivative without Singular Fractional Derivatives with Mittag-Leffler Kernel. In Fractional Derivatives with Mittag-Leffler Kernel; Springer: Cham, Switzerland, 2019 ; p. 293.

4. Kaddachi, N.; Jeribi, A.; Krichen, B. Fixed point theorems of block operator matrices on Banach algebras and an application to functional integral equations. Math. Methods Appl. Sci. 2013, 36, 659-673. [CrossRef]

5. Atkinson, F.V.; Langer, H.; Mennicken, R.; Shkalikov, A.A. The essential spectrum of some matrix operators. Math. Nachr. 1994, 167, 5-20. [CrossRef] 
6. Damak, M.; Jeribi, A. On the essential spectra of some matrix operators and application. Electron. J. Differ. Equ. 2007, 11, 1-16.

7. Amar, A.B.; Jeribi, A.; Krichen, B. Fixed Point Theorems for Block Operator Matrix and an Application to a Structured Problem Under Boundary Conditions of Rotenberg's Model type. Math. Slovaca 2014, 64, $155-174$. [CrossRef]

8. Kilbas, A.A.; Srivastava, H.M.; Trujillo, J.J. Theory and Applications of Fractional Differential Equations; Elsevier: Amsterdam, The Netherlands, 2006.

9. Chalishajar, D.; Kumar, A. Existence, uniqueness and Ulam's stability of solutions for a coupled system of fractional differential equations with integral boundary conditions. Mathematics 2018, 6, 96. [CrossRef]

10. Darwish, M.A.; Sadarangani, K. On generalized coupled fixed points with applications to the solvability of coupled systems of nonlinear quadratic integral equations. Fixed Point Theory 2018, 19, 527-544. [CrossRef]

11. Hashem, H.H.G.; El-Sayed, A.M.A. Solvability of coupled systems of fractional order integro-differential equations. J. Indones. Math. Soc. 2013, 19, 111-121. [CrossRef]

12. Shah, K.; Wang, J.; Khalil, H.; Khan, R.A. Existence and numerical solutions of a coupled system of integral BVP for fractional differential equations. Adv. Differ. Equ. 2018, 2018, 149. [CrossRef]

13. Cui, Y.; Sun, J. On existence of positive solutions of coupled integral boundary value problems for a nonlinear singular superlinear differential system. Electron. J. Qual. Theory Differ. Equ. 2012, 2012, 1-13. [CrossRef]

14. El-Sayed, A.M.A.; Hashem, H.H.G.; Ziada, E.A.A. Picard and Adomian Methods for coupled systems of quadratic integral equations of fractional order. J. Nonlinear Anal. Optim. Theory Appl. 2012, 3, 171-183.

15. Kumam, W.; Zada, M.B.; Shah, K.; Khan, R.A. Investigating a coupled Hybrid system of nonlinear fractional differential equations. Discret. Dyn. Nat. Soc. 2018, 2018, 5937572. [CrossRef]

16. Khalil, H.; Khan, R.A. New Operational Matrix of Integrations and Coupled System of Fredholm Integral Equations. Chin. J. Math. 2014, 2014, 146013. [CrossRef]

17. Shah, K. Coupled systems of boundary value problems for nonlinear fractonal differental equatons. J. Pure Appl. Math. 2018, 2, 14-17.

(C) 2019 by the authors. Licensee MDPI, Basel, Switzerland. This article is an open access article distributed under the terms and conditions of the Creative Commons Attribution (CC BY) license (http:/ / creativecommons.org/licenses/by/4.0/). 